\title{
Overview of Interference Situation and Mitigation Techniques for LDACS1
}

\author{
Ulrich Epple, Michael Schnell, German Aerospace Center (DLR), Germany
}

\begin{abstract}
LDACS1 is the broadband candidate technology for the future L-band digital aeronautical communications system. As unused spectrum is very scarce in the L-band, LDACS1 pursues the approach to make use of the gaps between adjacent channels used by the distance measuring equipment to meet the capacity requirements of a new aeronautical data link. This is a challenging approach as the power of DME signals is well above the LDACS1 received power in many scenarios. In addition, further legacy system, operating in the L-band might affect LDACS1. In this paper, we will characterize the different sources of interference and derive scenarios for their influence onto LDACS1. We will also distinguish between the situations in the forward, i.e. at an airborne receiver, and in the reverse link, corresponding to a ground station receiver. Next we will have a look onto detecting interference pulses which is a challenging task for LDACS1, as it uses orthogonal frequency division multiplexing modulation, which leads to remarkable fluctuations of the useful signal power itself. The efficiency of the proposed interference detection will be confirmed by realistic simulations. Based on the investigated detection capabilities, we will finally assess, by means of bit-error rate simulations, how the different types of interference influence the LDACS1 performance if a basic interference mitigation algorithm is applied.
\end{abstract}

\section{Introduction}

Currently, Air-Traffic Management (ATM) is undergoing an extensive modernization process within SESAR [1] and NextGen [2] in Europe and the US, respectively. This includes also the reorganization of aeronautical communications within the future communication infrastructure (FCI) [3] jointly carried out by Eurocontrol and the FAA. For air-to-ground communications, the L-band has been allocated and currently two candidates systems for superseding the analogue voice communication system in the VHF band are taken into consideration. The first candidate, L-band Digital Aeronautical
Communications System type 1 (LDACS1) is a broadband system employing Orthogonal FrequencyDivision Multiplexing (OFDM) as modulation. LDACS1 has been designed as a combination of P34 (TIA 902 standard) [4] and the Broadband Aeronautical Multi-carrier Communications (BAMC) system [5], [6]. It is designed as a FrequencyDivision Duplex (FDD) system. LDACS2 is a narrowband single-carrier system utilizing TimeDivision Duplex (TDD) as duplex scheme. This system is a derivative of the L-band Digital Link (LDL) and the All-purpose Multi-channel Aviation Communication System (AMACS).

LDACS1, which is the topic of this paper, is intended to be operated in the aeronautical part of the L-band (960-1164 MHz). The frequency band is already utilized by different legacy systems. This includes aeronautical navigation aids such as the distance measuring equipment (DME) or the military Tactical Air Navigation (TACAN) system as well as communication systems like the military Multifunctional Information Distribution System (MIDS) or the Joint Tactical Information Distribution System (JTIDS). Several fixed channels are allocated for the Universal Access Transceiver (UAT) at 978 $\mathrm{MHz}$ and for Secondary Surveillance Radar (SSR)/Airborne Collision Avoidance System (ACAS) at 1030 and $1090 \mathrm{MHz}$.

Due to these systems, free spectral resources are scarce and difficult to allocate in the L-band. For providing a sufficiently high capacity, LDACS1 intends to employ the spectral gaps between two adjacent channels assigned to the DME system as an inlay system. The small frequency separation of the two systems makes the design of the physical layer especially challenging. Furthermore, the design of the physical layer has to allow for alternatively deploying L-DACS1 without inlay in unused parts of the Lband.

To guarantee a reliable LDACS1 transmission, the system has to take countermeasures against the different sources of interference, such as presented in [7] or [8]. However, these methods require a reliable detection of the interference pulses. This topic was 
not addressed in detail in the literature yet. In [7] a perfect detection of interference was assumed, while in [8] only power detection was applied. In [9], the problem was addressed and an algorithm was proposed, but no quantitative results confirming the efficacy were given. In our paper, we propose two algorithms to detect the interference, which exploit the special characteristics of the DME interference in time- and frequency-domain.

The remainder of this paper is organized as follows. After a brief overview of the key LDASC1 physical layer parameters, we will characterize the different sources of interference, LDACS1 is exposed to. Special emphasis is put on the influence of the different systems onto LDACS1, given in terms of its duty-cycles at the LDACS1 receiver input. Next, we will present different algorithms for detecting interference pulses within the received signal. Their performance will be evaluated by means of simulations. For assessing the performance, we introduce two new metrics. Having confirmed the efficacy of the algorithms, we will further investigate the influence of the different sources of interference onto LDACS1 by means of bit-error-rate (BER) simulations. In the last section, conclusions are drawn and a short outlook for further work will be given.

\section{System Parameters}

In this section, we will give a short overview of the LDACS1 system, focusing on the system characteristics being relevant for the inlay approach and the robustness against interference. For more detailed information, please refer to the LDACS1 system specification [10] and [11].

\section{Main System Capabilities}

LDACS1 is intended to operate in the lower part of the L-band (960-1164 MHz). It is designed as an FDD system, which enables a Ground Station (GS) to transmit continuously at a certain frequency, while the Airborne Stations (AS) transmit at the same time but at a different frequency. For LDACS1, the frequency spacing between Forward Link (FL, from GS to AS) and the Reverse Link (RL, from AS to GS) is proposed to be $63 \mathrm{MHz}$.

For the LDACS1 deployment in the L-band different scenarios are possible. The most challenging approach is the inlay scenario where the LDACS1 channels with a bandwidth of approximately $500 \mathrm{kHz}$ are placed in between the existing DME channel grid of $1 \mathrm{MHz}$ with an offset of $500 \mathrm{kHz}$ to the DME center frequencies, as explained in the next section. This approach allows an LDACS1 deployment without changing existing DME assignments. For the inlay scenario the frequency range from 985.5 to $1008.5 \mathrm{MHz}$ is foreseen for the FL whereas the RL should be placed in the frequency range from 1048.5 to $1071.5 \mathrm{MHz}$. This choice minimizes the mutual interference between LDACS1 and other L-band systems, mainly SSR Mode S and UAT.

The LDACS1 signal is a multi-carrier signal, based on OFDM technology. The FL is a continuous OFDM transmission while the RL is based on Orthogonal Frequency-Division Multiple-Access / Time-Division Multiple-Access (OFDMA-TDMA) bursts assigned to different users on demand, which enables aircraft to adopt their duty-cycle and the allocation of different subcarriers according to the interference conditions. Another feature which is integrated into LDACS1 to adapt the throughput to the interference conditions is Adaptive Coding and Modulation (ACM), where different coding rates and the modulation schemes are supported. For strong interference, the most robust setting is QPSK modulation and a concatenated coding scheme with a rate $1 / 2$ convolutional encoder and a rate 0.9 ReedSolomon encoder.

\section{Selected Parameters}

The channel bandwidth of $498.05 \mathrm{kHz}$ is used by an OFDM system with 50 subcarriers, resulting in a subcarrier spacing of $9.765625 \mathrm{kHz}$. This subcarrier spacing was chosen as a trade-off between a high spectral efficiency and an acceptable inter-carrierinterference (ICI) caused by Doppler shifts of up to $1.25 \mathrm{kHz}$, typically occurring in the aeronautical environment.

For OFDM modulation, a 64-point FFT is used. The total FFT bandwidth comprising all subcarriers is $625.0 \mathrm{kHz}$. Besides the 50 subcarriers used for transmission, the 64 subcarriers comprise one direct current (DC) subcarrier as well as seven empty subcarriers at the left edge of the spectrum and six at the right edge, serving as guard bands. Exemplary, a FL Data/CC frame is depicted in Figure 1. 


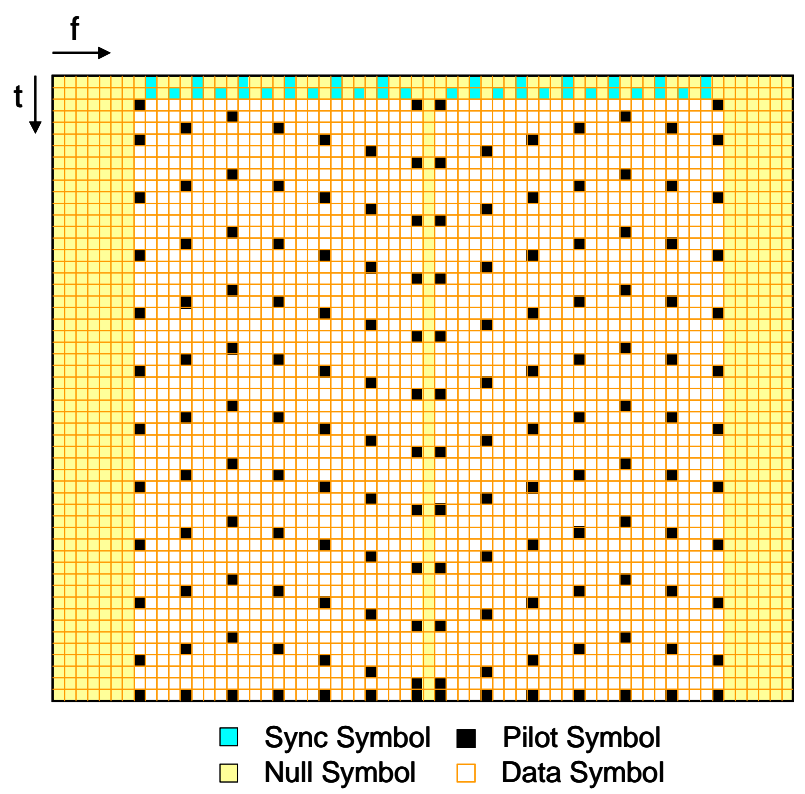

Figure 1. Structure of Data/CC Frame

According to the subcarrier spacing, one OFDM symbol has a duration of $102.4 \mu \mathrm{s}$. Each OFDM symbol is extended by a cyclic prefix of $17.6 \mu \mathrm{s}$, comprising a guard interval of $4.8 \mu$ s as well as 12.8 $\mu \mathrm{s}$ for transmit windowing. The guard interval provides resistance to inter-symbol interference caused by multipath effects. Transmit windowing leads to a reduction of the out-of-band radiation. This results in a total OFDM symbol duration of $120 \mu \mathrm{s}$ The main L-DACS1 OFDM parameters are listed in Table 1.

Table 1. LDACS1 OFDM Parameters

\begin{tabular}{|l|l|}
\hline Parameter & Value \\
\hline Effective bandwidth (FL or RL) & $498.05 \mathrm{kHz}$ \\
\hline Subcarrier spacing & $9.765625 \mathrm{kHz}$ \\
\hline Used subcarriers & 50 \\
\hline FFT length & 64 \\
\hline OFDM symbol duration & $102.4 \mu \mathrm{s}$ \\
\hline Cyclic prefix & $17.6 \mu \mathrm{s}$ \\
$\quad \quad$ guard time & $4.8 \mu \mathrm{s}$ \\
$-\quad$ windowing time & $12.8 \mu \mathrm{s}$ \\
\hline Total OFDM symbol duration & $120 \mu \mathrm{s}$ \\
\hline
\end{tabular}

\section{Framing Structure}

OFDM symbols are organized into LDACS1 frames. Depending on their functionality, different frame types are distinguished. The frames are arranged into Multi-Frames (MF) and Super-Frames (SF). The structure of a SF is depicted in Figure 2.

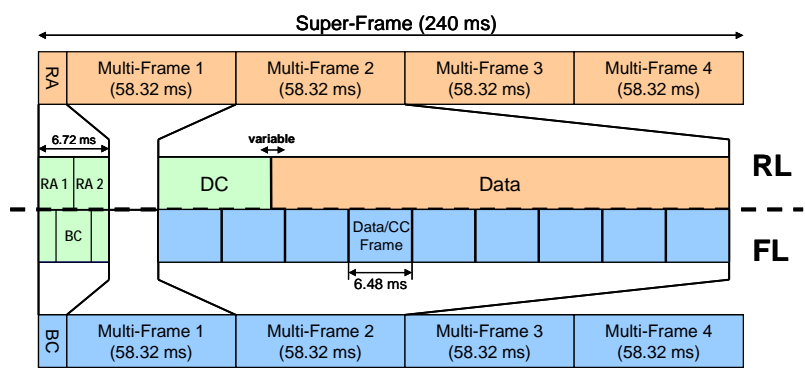

Figure 2. Super-Frame Structure

One SF contains of one Broadcast (BC) Frame in the FL and of one Random Access (RA) Frame in the RL, respectively, and of four MF in both, FL and RL. Each MF itself contains nine Data/CC frames in the FL and one Dedicated Control (DC) segment and one data segment in the RL.

\section{Characterization of Interference}

\section{Distance Measuring Equipment}

When LDACS1 is deployed as an inlay system between adjacent DME channels, DME signals represent the most severe interference towards LDACS1.

Mathematically, a DME signal consists of pairs of Gaussian shaped pulses which are described by

$$
p(t)=e^{-\alpha t^{2} / 2}+e^{-\alpha(t-\Delta t)^{2} / 2} .
$$

The parameter $\Delta t$ denotes the spacing of the pulses and depends on the certain mode of the DME station. The parameter $\alpha$ characterizes the width of one pulse. The used value $\alpha=4.5 \cdot 10^{11} \cdot 1 / \mathrm{s}^{2}$ leads to a width of $3.5 \mu$ s at $50 \%$ of the maximum amplitude. Figure 3 clarifies the shape of one DME pulse pair and the given parameters. 


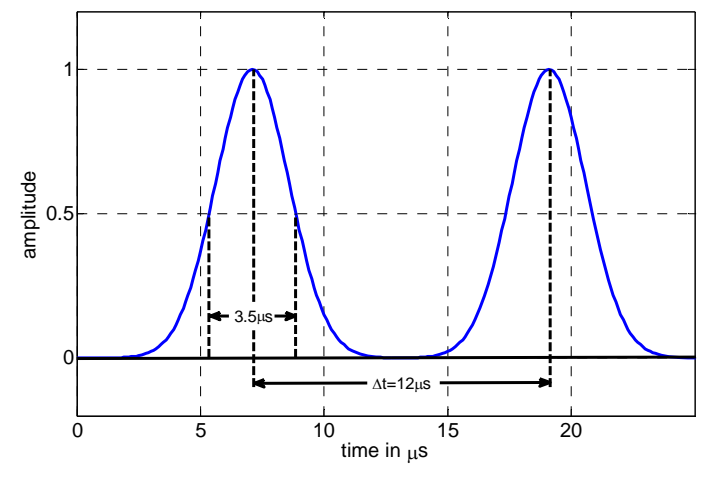

Figure 3. DME Pulse Pair in the Time Domain

Each DME transponder, i.e. GS transmits at a fixed center frequency, at a $1 \mathrm{MHz}$ channel grid in the range from $960-1215 \mathrm{MHz}$. The system is used for navigation purposes by calculating the distance between the GS and the AS. Therefore, a DME interrogator at an AS sends a request pulse pair and the DME interrogator replies after a fixed delay at a fixed frequency offset of $\pm 63 \mathrm{MHz}$, depending on the transmit frequency and the mode. A DME is usually coupled with a VOR device [12], which then allows, together with using knowledge about the altitude, the determination of the current position of the AS. For $\mathrm{X}$ mode, the interrogator and the transponder transmit both with $\Delta \mathrm{t}=12 \mu \mathrm{s}$. In $\mathrm{Y}$ mode, the interrogator transmits with $\Delta \mathrm{t}=36 \mu \mathrm{s}$, while the transponder answers with $\Delta \mathrm{t}=30 \mu \mathrm{s}$. Note that the two other DME modes, $\mathrm{W}$ and $\mathrm{Z}$ mode are neglected in our case, since they are only used for DME/P (precision), which is employed for the microwave landing system (MWS), while we are focusing on the En-route domain.

A Gaussian shaped pulse also leads to a Gaussian shaped spectrum. Since pulses are occurring pair-wise, the spectrum exhibits fades. Figure 4 shows exemplarily a $4 \mathrm{MHz}$ detail of the Lband, comprising signals from four adjacent DME channels.

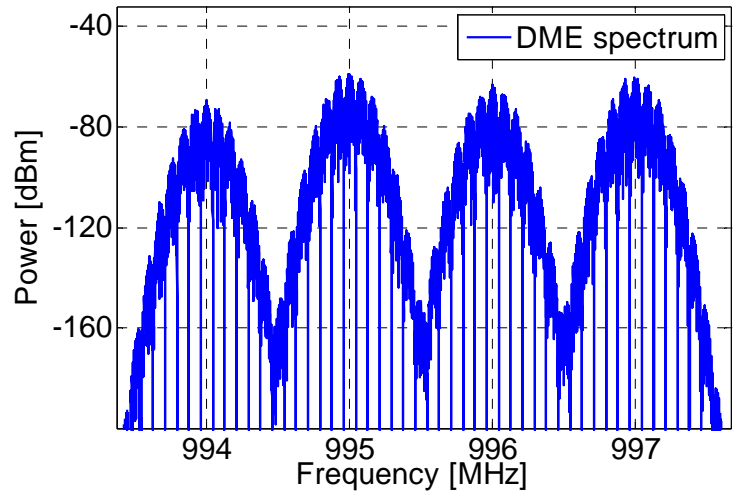

Figure 4. Spectrum of Four Adjacent DME Channels

The transmission rate is given by the number of pulse pairs per second (ppps). A DME interrogator distinguishes between search and track mode. In search mode, it may transmit up to 150 ppps. When a connection to a transponder could be established, the rate decreases to 30 ppps. The squitter rate of DME transponders depend on the number of aircraft, it has to serve. In maximum, 2700 ppps is reached [13].

The typical transmit peak power of DME on-ground transponder can be given by $1 \mathrm{~kW}$, corresponding to $60 \mathrm{dBm}$. Airborne interrogators transmit with 63 $\mathrm{dBm}$ [14]. The maximum allow EIRP is $70 \mathrm{dBm}$, taking antenna gains and cable losses into account.

\section{Tactical Air Navigation}

TACAN is a military navigation system, based on the DME system. In contrast to DME, it is capable to calculate not only the distance between a GS and an AS, but also the direction of the transponder signal at the aircraft. Concerning the interference onto LDACS1, TACAN differs only in the squitter rate, which is augmented to 3600 ppps for TACAN transponder since a TACAN transponder transmits additional 900 specially coded ppps. The GS transmit peak power is $3 \mathrm{~kW}$, i.e. $64.8 \mathrm{dBm}$.

For estimating the received power at an LDACS1 receiver, an over-flight of a TACAN GS at flight level 450 is considered in [15]. This corresponds to a vertical distance of 7.4 nautical miles and leads to a free space loss of $\approx-115 \mathrm{~dB}$. In this case, the received DME power is more than 50 $\mathrm{dB}$ above the required LDACS1 sensitivity of $\mathrm{S}_{0}=-102.83 \mathrm{dBm}[10]$ and one can conclude that the 
impact of DME interference onto LDACS1 is governed by the duty-cycle but not by the interference power, as in most use cases the interference power is well above the useful signal power. For a typical scenario in the FL, we assume that an airborne LDACS1 receiver is exposed to interference from one DME/TACAN station at a frequency offset of $0.5 \mathrm{MHz}$. The pulse duration of $3.5 \mu \mathrm{s}$ and the pulse raise- and decay-time of $2.5 \mu \mathrm{s}$, respectively, lead to a duty-cycle of

$$
D_{\text {TACAN,typ }}=\frac{2 \cdot(3.5 \mu \mathrm{s}+2.5 \mu \mathrm{s}+2.5 \mu \mathrm{s})}{1 \mathrm{~s}} \cdot 3600=6.1 \% \text {. }
$$

In the worst-case, it was identified that up to three TACAN stations comprising a high received power might be active in the adjacent channels [7]. Since the different interference pulses might overlap, the cumulated duty-cycle is calculate according to

$$
D_{\text {TACAN,wc }}=1-\left(1-D_{\text {TACAN,typ }}\right)^{3}=17.2 \% \text {. }
$$

In the RL, the interference situation is different, since we can assume that many aircraft are within the radio horizon of an LDACS1 GS receiver. In this case, also many interference pulses will occur with a received power in the range of the LDACS1 received power. In [15], the worst-case interference is given in terms of a probability density function (pdf) of the received interference power. The squitter rate was derived to be 8334 ppps leading to a duty-cycle of

$$
D_{\text {DME,RL }}=\frac{2 \cdot(3.5 \mu \mathrm{s}+2 \cdot 2.5 \mu \mathrm{s})}{1 \mathrm{~s}} \cdot 8334=14.2 \% \text {. }
$$

However, this duty-cycle would assume that the power of all interference pulses is well above the useful signal power. In reality, also according to the derived pdf, many interference pulses comprise only low power, leading to shorter distortions.

The interference from other DME GSs onto an LDACS1 GS can be avoided by an effective placement of the GSs and its antennas.

\section{Co-site Interference}

Co-site interference occurs due to the insufficient antenna isolation between legacy system transmit antennas and the LDACS1 receive antenna, equipped on-board. Obviously, this is no matter at the GS, where a sufficient high antenna isolation is guaranteed. Although the legacy systems, namely
DME, SSR, and UAT, transmit at frequencies well separated from the LDACS FL center frequencies, the power of the occurring broadband noise might be well above the useful signal power at the LDACS1 aircraft receiver. In [16], the expected interference power of the three systems at the LDACS1 receiver was derived. Taken the spectral masks of the different systems and a realistic antenna isolation of $35 \mathrm{~dB}$ into account, the broadband noise power, which is summarized Table 2, is well above the required LDACS1 sensitivity of $\mathrm{S}_{0}=-102.83 \mathrm{dBm}$.

Table 2. Co-site Noise Power

\begin{tabular}{|c|c|}
\hline System & Broadband Noise Power $P_{\text {noise }}$ \\
\hline DME & $-23.8 \mathrm{dBm}$ \\
\hline UAT & $-33.5 \mathrm{dBm}$ \\
\hline SSR & $-36 \mathrm{dBm}$ \\
\hline
\end{tabular}

As this interference occurs within the whole LDACS1 bandwidth and features no special spectral shape, the complete useful signal is assumed to be lost when coinciding with co-site interference.

Next we will investigate the duty-cycle of the different co-site systems. For DME, the maximum transmission rate is 150 ppps, as given above. The pulse duration of $3.5 \mu$ s and the pulse raise- and decay-time of $2.5 \mu \mathrm{s}$, respectively, lead to a dutycycle of

$D_{\text {co-site,wc }}=\frac{2 \cdot(3.5 \mu \mathrm{s}+2 \cdot 2.5 \mu \mathrm{s})}{1 \mathrm{~s}} \cdot 150=0.26 \%$.

Fortunately, most of the time the DME will work in track mode, lowering the duty-cycle to a typical value of $0.05 \%$.

A UAT frames last $1 \mathrm{~s}$. Within this frame, an aircraft can transmit one automatic dependant surveillance broadcast (ADS-B) message, lasting $276 \mu$ s for a short ADS-B and $420 \mu$ s for a long ADS-B message. This leads to a maximum duty-cycle of $\mathrm{D}_{\mathrm{UAT}, \mathrm{wc}}=$ $0.42 \%$.

Finally, the duty-cycle of SSR shall be given. This is not trivial, as different radar types like TCAS, mode $\mathrm{A} / \mathrm{C}$, mode $\mathrm{S}$ or the extended squitter are comprised within the SSR system. In [13] a typical and a worst- 
case scenario for the occurrence of SSR pulses have been defined, which are summarized in Table 3.

Table 3. SSR Transmit Profiles

\begin{tabular}{|c|c|c|c|}
\hline Radar type & $\begin{array}{c}\text { Rate/1s } \\
\text { (typical) }\end{array}$ & $\begin{array}{c}\text { Rate/1s } \\
\text { (worst- } \\
\text { case) }\end{array}$ & $\begin{array}{c}\text { Transmit } \\
\text { frequency }\end{array}$ \\
\hline TCAS & 5 & 7 & $1090 \mathrm{MHz}$ \\
\hline $\begin{array}{c}\text { Mode S } \\
\text { (Roll call) }\end{array}$ & 6 & 9 & $1090 \mathrm{MHz}$ \\
\hline $\begin{array}{c}\text { Mode S } \\
\text { (All Call) }\end{array}$ & 15 & 40 & $1090 \mathrm{MHz}$ \\
\hline $\begin{array}{c}\text { Extended } \\
\text { Squitter }\end{array}$ & 4 & 6 & $1090 \mathrm{MHz}$ \\
\hline $\begin{array}{c}\text { Short } \\
\text { squitter }\end{array}$ & 1 & 1 & $1090 \mathrm{MHz}$ \\
\hline $\begin{array}{c}\text { TCAS } \\
\text { (Mode S) }\end{array}$ & 7 & 12 & $1030 \mathrm{MHz}$ \\
\hline
\end{tabular}

One should note that mode A/C is excluded, as it is assumed that at the time when LDACS1 will be deployed, surveillance will not rely anymore on mode A/C. The maximum pulse duration of SSR pulses is $112 \mu$ s for the long mode $S$ pulse [17]. This leads to a typical duty-cycle of

$D_{\mathrm{SSR}, \mathrm{typ}}=\frac{112 \mu \mathrm{s}}{1 \mathrm{~s}} \cdot(5+6+15+4+1+7)=0.43 \%$,

and a worst-case duty-cycle of $\mathrm{D}_{\mathrm{SSR}, \mathrm{wc}}=0.84 \%$.

\section{Joint Tactical Information Distribution System}

The joint tactical information distribution system (JTIDS) is a digital military radio system, mainly used by the NATO. It is based on TDMA and provides a jam-resistant mode, by employing frequency hopping in a pseudo-random way. Therefore it uses 51 frequencies in the frequency band $960-1215 \mathrm{MHz}$, excluding areas around the SSR frequencies 1030 and $1090 \mathrm{MHz}$. The 51 frequencies have a $3 \mathrm{MHz}$ spacing. In Figure 5, the frequency hopping principle is depicted for a short time period.

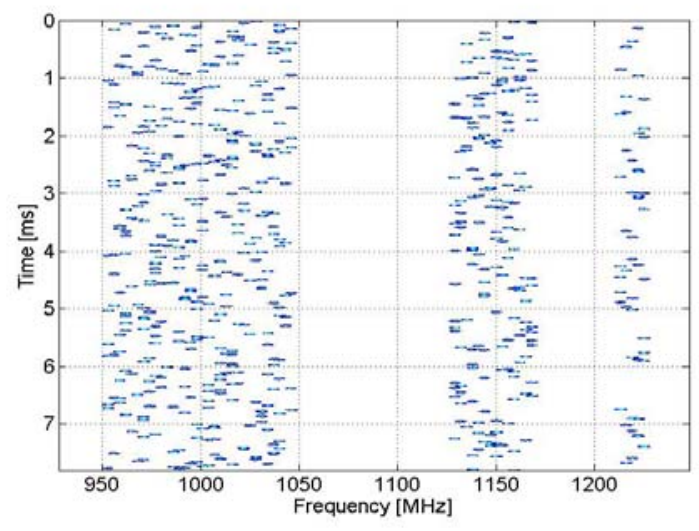

Figure 5. JTIDS Frequency Hopping Scheme

The TDMA framing is composed out of frames with a duration of $12 \mathrm{~s}$. One frame itself is divided into 1536 slots, which again consist of sequences of either 72, 258, or 444 pulses. The frequency hopping is applied from pulse to pulse with a pulse duration of $13 \mu \mathrm{s}$, comprising a $6.4 \mu$ s active pulse time with a trapezoid pulse shape and $6.6 \mu$ s guard time. The JTIDS spectrum is flat within a bandwidth of \pm $3 \mathrm{MHz}$. According to the spectral mask, depicted in Figure 6 , at $\pm 5 \mathrm{MHz}$, an attenuation of $-23 \mathrm{~dB}$ demanded.

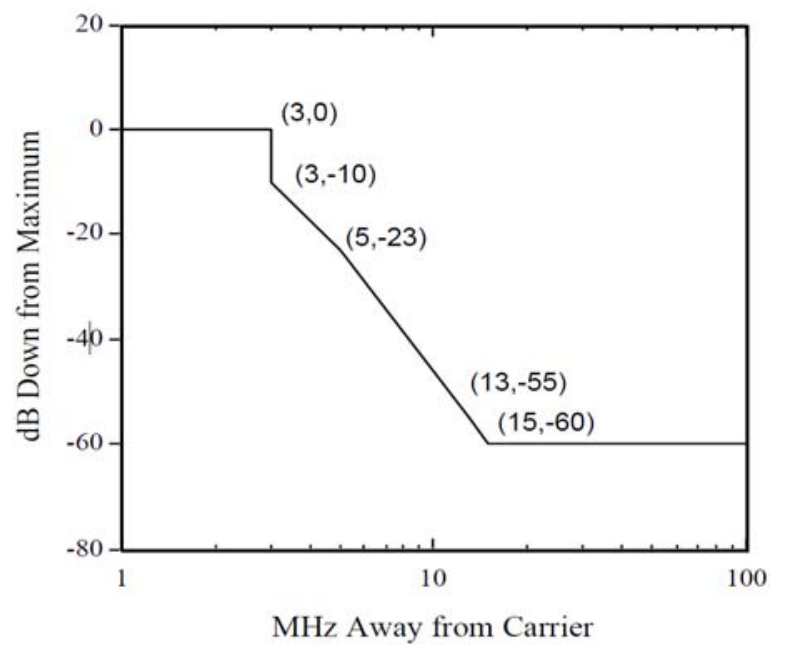

Figure 6. JTIDS Spectral Mask

In principle, the maximum allowed transmit power of a JTIDS transmitter is $1000 \mathrm{~W}$. However, most national Frequency Clearance Agreements (FCA) limit this power to $200 \mathrm{~W}(53 \mathrm{dBm})$, which is below the maximal permitted DME/TACAN transmit power [13]. 
For characterizing the influence onto LDACS1, we have to define the duty-cycle at a certain receive frequency. In total, the JTIDS framing leads to a maximum number of 56832 pulses per second. For a JTIDS network, the maximum time slot duty factor (TSDF) for one user is $50 \%$, which means that only every second time slot is utilized, lowering the number of pulses to 28416 per second. Due to the frequency hopping, only a fraction of these pulses will affect the chosen LDACS1 frequency. When keeping the spectral mask and the maximal allow transmit power in mind, we assume that in a typical use case, i.e. medium/large range between the JTIDS transmitter and the LDACS1 receiver, three JTIDS hopping frequencies will affect the LDACS1 signal, and the receive power of a larger frequency spacing will be below the sensitivity level of LDACS1. In addition, typically a TSDF of $50 \%$ is assumed, leading to a total duty-cycle of

$D_{\text {JTIDS,typ }}=\frac{6.4 \mu \mathrm{s}}{1 \mathrm{~s}} \cdot \frac{3}{51} \cdot 28416=1.1 \%$.

For the worst-case, we assume that the receive power of the JTIDS signal is significantly above the LDACS1 receive power. In this case, all hopping frequencies within $\pm 13 \mathrm{MHz}$ around the LDACS1 center frequency, corresponding to nine hopping frequencies, will impair the reception of LDACS1. In combination with a worst-case TSDF of $100 \%$, we obtain a total duty-cycle of

$D_{\text {JTIDS }, \mathrm{wc}}=\frac{6.4 \mu \mathrm{s}}{1 \mathrm{~s}} \cdot \frac{9}{51} \cdot 56832=6.4 \%$.

\section{Detection of Interference}

The detection of the L-band interference, described in the previous section, is a challenging task for LDACS1, as this multi-carrier system is based on OFDM modulation. One of the main drawbacks of OFDM is the fluctuation of the signal amplitude, which leads to a high peak-to-average power ratio (PAPR). This puts high demands on the linearity of the transmit- and receive-amplifiers. In addition, the amplitude fluctuations complicate the detection of interference pulses, especially if their amplitude is in the range of the OFDM peaks. There are several proposals for lowering the PAPR [18]. For LDACS1, in all frame-types, the pilots are chosen to comprise a low PAPR itself, which decreases the PAPR of the whole OFDM signal. In addition, in the RL frames, two PAPR reduction subcarriers are inserted in each OFDM symbol. In contrary to the pilot symbols, the values of these subcarriers are not predefined, but calculated depending on the other modulated subcarriers in the respective OFDM symbol. This lowers the PAPR remarkably. In the FL, no additional PAPR reduction methods are deployed, as it is assumed that in LDACS1 GSs high-end amplifier are installed, reducing the difficulties with the linearity of the transmit amplifier.

The basic method for detecting interference is to compare the received signal power with a threshold. If the threshold is exceeded, the signal is detected as an interference pulse. This method is mainly suited for interference pulses with a power significantly above the useful signal, such as strong JTIDS and DME pulses and interference, exhibiting no special shape in time or frequency domain such as broadband noise, caused by co-site interference. The threshold has to be chosen as a trade-off between detecting as much interference as possible, and discarding as few useful signal as possible.

For DME signals with an interference power in the range of the LDACS1 receive power, as it may occur in both, FL and RL for large distances between DME station and LDACS1 receiver, we will present two methods. They employ on the one hand the repetitive structure of DME pulse pairs, and on the other hand the Gaussian shaped interference spectrum.

For employing the repetitive structure of DME pulses, we propose a correlation of parts of the received signal $y(d)$ with shifted parts. The correlation $P(d)$ is chosen according to the length of DME pulses and the shifting according to the distance of the two DME pulses of one pulse pair.

$$
\begin{aligned}
& P(d)=\sum_{m=0}^{N_{\text {corr }}-1}\left(y^{*}(d+m) \cdot y\left(d+m+N_{\text {diff }}\right)\right), \\
& \text { with } \\
& N_{\text {corr }}=\frac{3.5 \mu \mathrm{s}+2 \cdot 2.5 \mu \mathrm{s}}{t_{\text {samp }}}, \\
& N_{\text {diff }}=\frac{\Delta t}{t_{\text {samp }}},
\end{aligned}
$$


and $t_{\text {samp }}$ being the sampling time. This correlation is normalized by the power of the correlated signal $N(d)$, leading to a maximal correlation value of one.

$N(d)=\frac{1}{2} \sum_{m=0}^{N_{\text {corr }}-1}\left(|r(d+m)|^{2}+\left|r\left(d+m+N_{\text {diff }}\right)\right|^{2}\right)$,

$M_{\text {aux }}(d)=\frac{|P(d)|^{2}}{(N(d))^{2}}$.

To avoid peaks from signal parts which are correlated but possess a low interference power, the correlation metric is finally multiplied with the power of the received signal, leading to the following term $M(d)$ for the correlation metric

$M(d)=|y(d)|^{2} \cdot M_{\text {aux }}(d)$.

Next, a rather high threshold is defined to detect the peaks of the DME pulses. For detecting all samples of the DME pulses, a second, lower threshold is defined and all received samples in the vicinity of detected peaks, which are above this second threshold, are considered as interference. Note that also all samples, shifted by the pulse distance are interpreted as interference, as they also contribute to the metric and DME signals always occur pulse-wise. This method is expected to reduce the number of false alarms, as OFDM signal peaks are not supposed to occur pulse-wise and the correlation will neglect single peaks. In addition, the introduction of two different thresholds will reduce the number of false alarms peaks but preserving the ability to detect complete DME pulses.

An additional approach exploits the spectral shape of the DME signals and can be applied when employing LDACS1 as an inlay system. In this case, the distortion power is maximal at the edges of the LDACS1 spectrum and slopes down to the center frequency. For separating the interference signal from the useful signal, we will high- and low-pass filter the signal, as depicted in Figure 7.

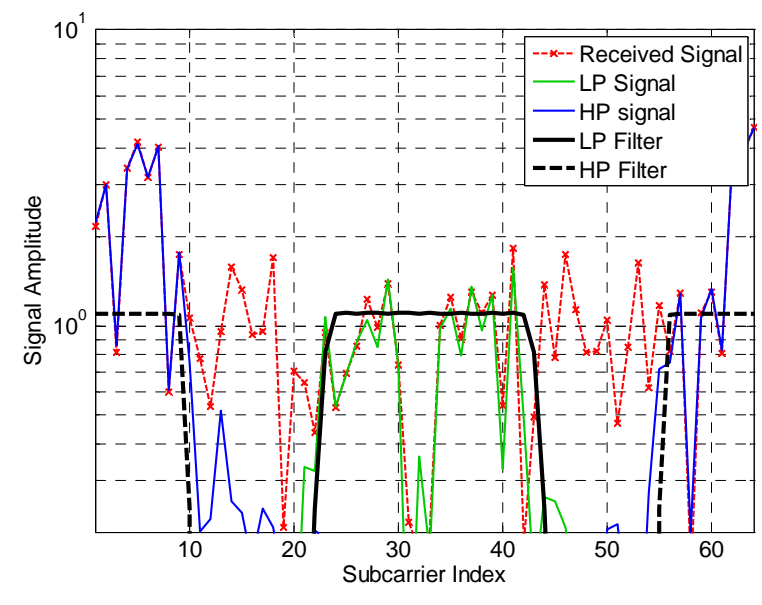

\section{Figure 7. Principle of Low- and High-Pass Filtering}

The low-pass signal contains no interference contributions thus we can estimate the useful signal energy based on this signal. The high-pass signal comprises besides the interference, only the LDACS1 signal at the edge subcarriers, lowering the PAPR of the high-pass signal remarkably. After having normalized the signal power by the filter bandwidth, we are able to estimate the interference power, by subtracting the power of the low-pass signal, i.e. the LDACS1 signal power, from the high-pass signal power, which comprises both signals.

This is followed again by a two-step threshold detection, as it has been described for the correlation method.

Figure 8 illustrates the beneficial influence of both methods for a received signal, which is affected by DME pulses with a power in the range of the useful signal. While for the received signal itself it is difficult to distinguish between OFDM signal peaks and interference, both methods lead to a much better highlighting of the interference. 


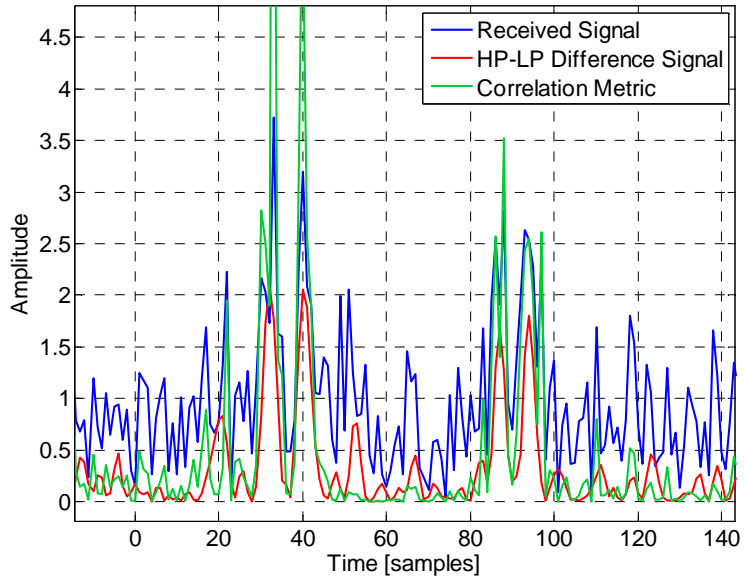

Figure 8. Metrics of Pulse Detection Methods

\section{Performance of Interference Detection}

In this section, the efficiency of the algorithms presented above shall be validated by means of simulations. Therefore, two metrics will be introduced to quantify the capabilities of the detection schemes. The first metric shall evaluate the ability to detect samples distorted by interference and is defined as the ratio of interference samples which are not detected by the respective algorithm and the total number of received samples. In addition it is also important to minimize the number of falsely detected interference. The second metric is defined by the number of falsely detected samples over the total number of received samples. One should note that it is only desirable to detect samples as interference, if the interference power compared to the useful signal power exceeds a certain threshold. For a lower interference power, the skipping of the useful signal is adverse to removing the interference. The optimal detection threshold depends on the subsequent interference mitigation. When applying pulse blanking [8], the optimal threshold was derived in [9]. For a more sophisticated pulse blanking compensation [7], the threshold is lower. We adopted these lower threshold, $\mathrm{T}_{\mathrm{PB}}=1.25$, as for LDACS1, it is intended to apply not only pulse blanking.

In Figure 9, the missed interference samples ratio is plotted versus the DME peak power $\mathrm{I}_{\mathrm{DME}}$ of one TACAN station with a squitter rate of $3600 \mathrm{ppps}$ and a frequency offset of $-0.5 \mathrm{MHz}$ for a fixed signalto-noise ratio (SNR) of $4 \mathrm{~dB}$. This power refers to the power at the receiver input and will be remarkably attenuated by receiver filtering. The signal power is assumed to be fixed at $\mathrm{P}_{\text {sig }}=-100 \mathrm{dBm}$, which is slightly above the sensitivity of $\mathrm{S}_{0}=-102.83 \mathrm{dBm}$. The $\mathrm{I}_{\mathrm{DME}}=-70 \mathrm{dBm}$ leads to interference peaks, which are slightly above the threshold $\mathrm{T}_{\mathrm{PB}}=1.25$, if the signal is normalized to $\mathrm{P}_{\mathrm{sig}}=0 \mathrm{dBm}$. The LDACS1 signal itself results from a FL transmission. In this case, the PAPR is even higher than in the RL, as no PAPR reduction subcarrier are inserted. For all three methods, the missed samples ratio decreases when the interference power increases, as in this case the distinction between the LDACS1 signal and the interference power becomes more and more easy. As expected, the simple power detection performs worst, followed by the correlation method and the high-pass low-pass filtering method. However, one should note that the latter can only be used for detecting DME pulses with $500 \mathrm{kHz}$ frequency offset, while the correlation method detects all received DME pulses. For all methods, the missed sample ratio is below $3 \%$, even for an interference power in the range of the OFDM signal power and a fairly low SNR of $4 \mathrm{~dB}$.

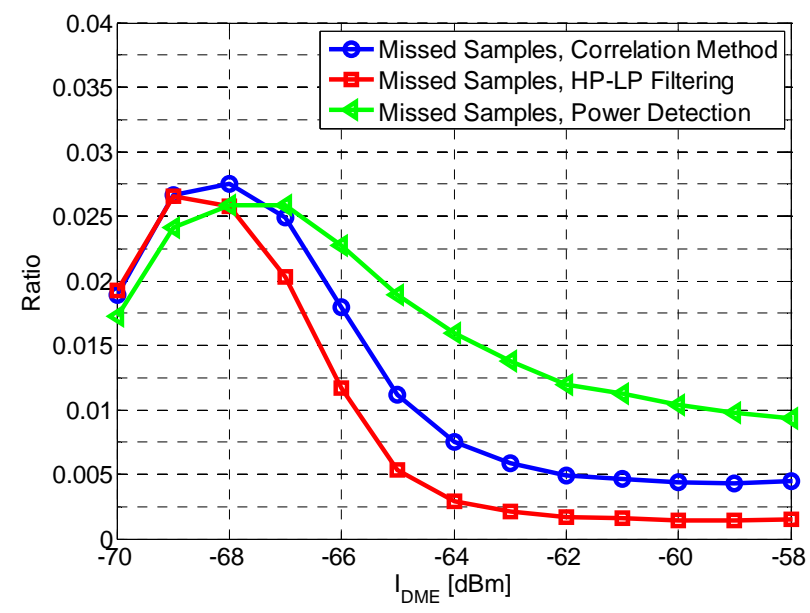

Figure 9. Missed Samples Ratio vs. $\mathbf{I}_{\mathrm{DME}}$ for SNR = $4 \mathrm{~dB}$

In Figure 10, the falsely detected interference ratio is plotted for the same parameter set as considered above. Again, the high-, low-pass filtering method performs best, while the power detection produces the most falsely detected samples due to the incapability to distinguish between interference and signal peaks. However, all three methods produce less than $2 \%$ of falsely detected samples and the filtering method even less than $1 \%$. 
Interestingly, the maximum number of falsely detected samples is at $I_{D M E}=-65 \mathrm{dBm}$, while the maximum number of missed interference samples occurs at $\mathrm{I}_{\mathrm{DME}}=-68 \mathrm{dBm}$, i.e. for much smaller interference pulses.

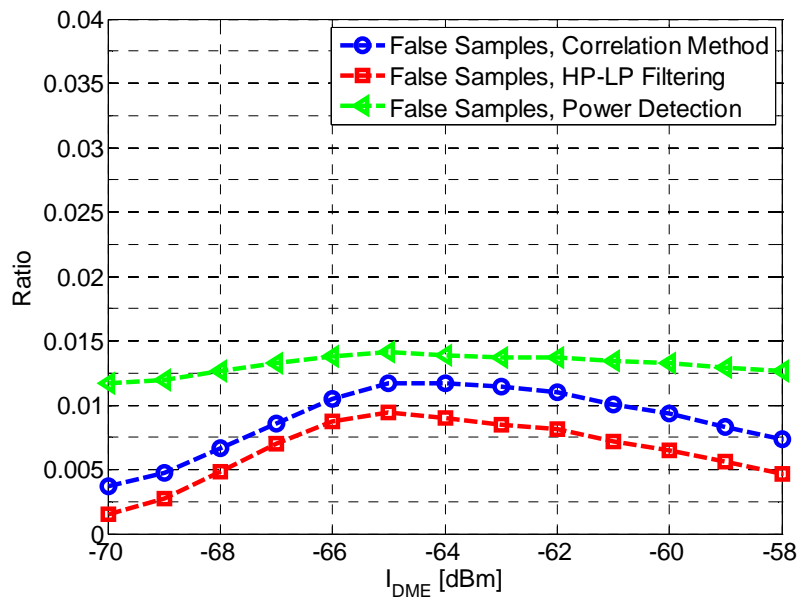

Figure 10. False Samples Ratio vs. $I_{D M E}$ for $S N R=$ $4 \mathrm{~dB}$

For this maximum of $\mathrm{I}_{\mathrm{DME}}=-68 \mathrm{dBm}$, we will also investigate the influence of the SNR onto the detection capabilities, as strong noise will also lead to undesired peaks in the received signal. In Figure 11, the missed and falsely detected samples ratios are plotted versus the SNR. The other parameters are the same as above. Interestingly, the performance of the high-, low-pass filtering method does not depend on the SNR, even for low SNR values, i.e. high noise peaks, the false detected samples ratio is below $1 \%$. In opposite, the power detection suffers remarkably from strong noise and produces many false detected samples. For low SNR, it also exhibits the smallest missed samples ratio, as the noise contribution raises many interference samples over the threshold. For increasing SNR values, all methods converge against similar missed and falsely detected samples ratios.

In summary one can state, that for moderate and high SNR values, all methods perform well. For small SNR values, especially if the interference power is in the range of the useful signal energy, more sophisticated interference detections like the correlation method and primarily the high-, low-pass filtering method can improve the detection performance remarkably, compared to the simple power detection and leads to a reliable detection.

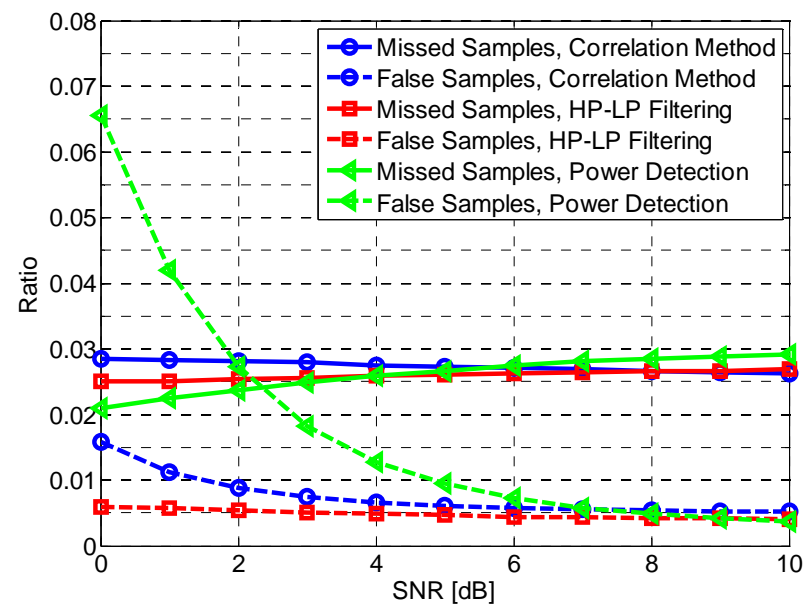

Figure 11. Missed and False Samples Ratio vs. $S N R$ for $I_{D M E}=-68 \mathrm{dBm}$

\section{Overall System Performance}

For assessing the impact of the different sources of interference onto LDACS1, BER simulations were performed. As the different interference detection algorithm work good, even for a low or medium interference power, in the following, a perfect detection of the interference is assumed. When applying in a first step pulse blanking for mitigating the interference, the interference is modeled as blanks in the received signal according to the different dutycycles, derived above. As we are only interested in the degradation of the system performance by interference, but not in the absolute system performance, only additive white Gaussian noise (AWGN), but no additional channel model is applied. Further, we will focus on the FL, as this is assumed to be the more challenging case, as co-site interference only occurs airborne. In addition, the most robust ACM setting, i.e. QPSK modulation and rate $1 / 2$ convolutional coding was chosen.

In Figure 12, the BER of the LDACS1 FL is plotted versus the SNR for the different interference sources separately. The co-site interference leads, even for the worst-case to no system degradation. Also a typical JTIDS employment does not degrade our system performance. The worst-case JTIDS scenario leads to a recognizable performance degradation, which is still very little. As expected, the most severe system degradation results from the DME/TACAN stations. Even a typical case, with one strong station in an adjacent channel leads to a 
system degradation of $1 \mathrm{~dB}$. In an unkind environment, the degradation is even above $3 \mathrm{~dB}$.

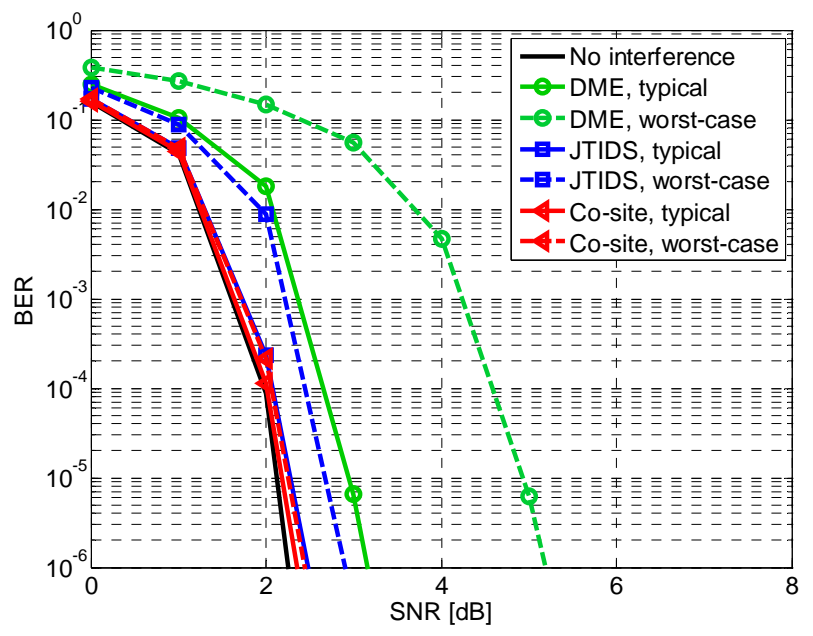

\section{Figure 12. BER vs. SNR for Different Interfering Systems}

For applying all interference sources jointly, the resulting BER is plotted versus the SNR in Figure 13 for both, typical and worst-case assumptions. In both cases, the performance degradation is governed by the DME interference. But while for a typical deployment, the performance is similar to the DME performance itself, for the worst-case assumption, the performance degrades additionally due to the other systems, leading to an overall system loss of $4 \mathrm{~dB}$. One should mention that for mitigating the interference, simply pulse blanking but no additional methods were employed. When applying a more sophisticated interference mitigation like in [7], a considerable performance re-gain is expected, indicating the feasibility of LDACS1, even under worst-case interference conditions.

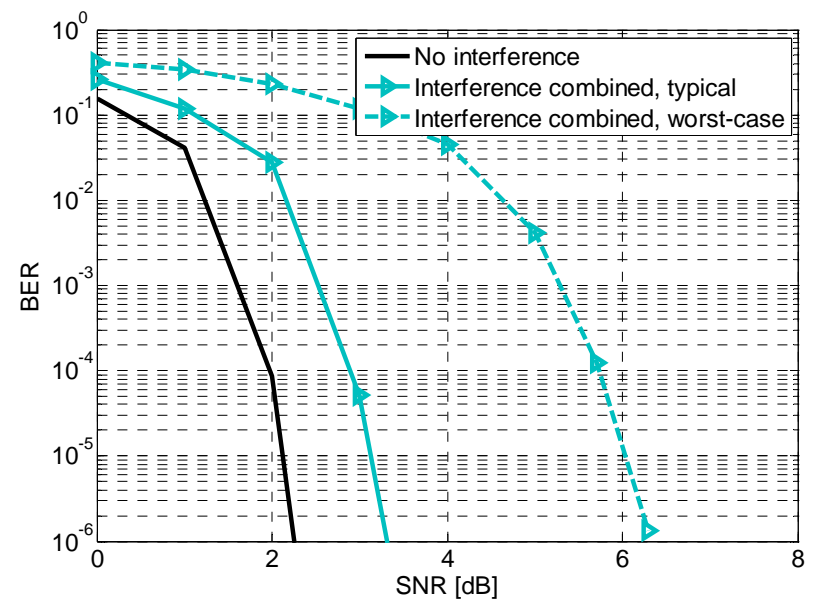

\section{Figure 13. BER vs. SNR for Combined Interference Scenario}

\section{Conclusion and Outlook}

In this paper, we addressed the interference situation, a future aeronautical communications system in the L-band is exposed to. We presented and characterized the different legacy system, operating in the L-band, in terms of their spectral characteristics, their transmit power and the dutycycle. For the candidate system LDACS1, we also derived typical and worst-case scenarios for their influence onto LDACS1.

Next we developed two algorithms for detecting interference, resulting from the DME system, which is superimposed with an LDACS1 signal. The feasibility of these algorithms is confirmed by means of realistic simulations, which show a superior performance, compared to a standard detection method.

Finally, the influence of the different interference systems onto LDACS1 was determined. Therefore bit-error rate simulations were carried out, assuming a reliable detection and blanking of the interference pulses. It turned out, that typical interference scenarios will just lead to a small performance degradation, only for worst-case assumptions, in particular DME interference will lead to a serious loss in performance.

For the future, additional bit-error rate simulation will be carried out, additionally comprising a sophisticated interference mitigation, a realistic channel model and estimation as well as real interference signals. Furthermore, the reverse link 
will be investigated, which was neglected in this paper, as the interference situation is less severe.

\section{References}

[1] http://www.sesarju.eu/

[2] http://www.faa.gov/nextgen/

[3] Framework for Spectrum Compatibility Analysis in L-Band for FCI technology Candidates, EUROCONTROL, Draft 1.0.

[4] TIA standard family TIA-902, 2002/2003.

[5] Schnell, Michael, S. Brandes, S. Gligorevic, C.-H. Rokitansky, M. Ehammer, Th. Gräupl, C. Rihacek, M. Sajatovic, 2008, "B-AMC - Broadband Aeronautical Multi-carrier Communications", 2008 Integrated Comm. Navigation and Surveillance Conf. (ICNS 2008), Bethesda, MD, USA.

[6] Rokitansky, Carl-Herbert, M. Ehammer, Th. Gräupl, M. Schnell, S. Brandes, S. Gligorevic, C. Rihacek, and M. Sajatovic, 2007, "B-AMC - A system for future Broadband Aeronautical MultiCarrier communications in the L-Band," 2007 Digital Avionics Systems Conference (DASC 2007), Dallas, TX, USA, pp. 4.D.2-1- 4.D.2-13.

[7] Brandes, Sinja, Ulrich Epple, Michael Schnell, 2009, Compensation of the Impact of Interference Mitigation by Pulse Blanking in OFDM Systems, Honululu, HI, USA, Proc. of IEEE Globecom 2009.

[8] Zhidkov, Sergey V., 2008, Analysis and Comparison of Several Simple Impulsive Noise Mitigation Schemes for OFDM Receivers, IEEE Transactions on Communications, vol. 56, no. 1, pp. 5-9.

[9] Brandes, Sinja, Michael Schnell, 2009, Interference Mitigation for the Future Aeronautical L-Band Communication System, 7th International Workshop on Multi-Carrier Systems \& Solutions (MC-SS 2009), Herrsching, Germany, Springer, pp. 375-384.
[10] Sajatovic, Miodrag, B. Haindl, M. Ehammer, Th. Gräupl, M. Schnell, U. Epple, S. Brandes, February 2009, "LDACS1 System Definition Proposal: Deliverable D2", Edition 1.0, Eurocontrol Study.

[11] EWA04-2-T1-D2, 2011, Insert Paper Title Here (No More Than 80 Characters), SESAR, Ed. 00.01.01.

[12] M. Kayton, W. Fried, "Avionics Navigation Systems”, Wiley, $2^{\text {nd }}$ edition, 1997

[13] EWA04-2-T1-D2, 2010, Updated Interference Scenarios for Existing L-band Systems, SESAR, Ed. 00.00.01.

[14] FCS - Framework for Spectrum Compatibility Analysis in L-band for FCI Technology Candidates, EUROCONTROL, Draft 1.0.

[15] B-AMC Deliverable D5, 2007, Expected BAMC System Performance, EUROCONTROL, Ed. 1.1 .

[16] EWA04-1-T1-D1, 2010, Preliminary Report on Interference Mitigation Techniques, SESAR, Ed. 00.00.01

[17] ICAO, 2002, Annex 10 Vol. 4, Third Edition.

[18] Han, Seung Hee, JAE HONG LEE, 2005, An Overview of Peak-to-Average power ratio reduction techniques for Multicarrier Transmission, IEEE Wireless Communications, pp. 56-65.

\section{Email Addresses}

ulrich.epple@dlr.de

michael.schnell@dlr.de

30th Digital Avionics Systems Conference October 16-20, 2011 